OPEN ACCESS Shama Prasada Kabekkodu, Manipal Academy of Higher Education, India

Reviewed by: Arsheed A. Ganaie, University of Minnesota Twin Cities, United States

Manjunath B. Joshi, Manipal Academy of Higher Education, India

*Correspondence: Yun-Hui Li 18909882737@163.com Wei-Neng Fu wnfu@cmu.edu.cn

Specialty section: This article was submitted to Cancer Genetics, a section of the journal

Frontiers in Oncology

Received: 19 August 2020 Accepted: 30 December 2020 Published: 18 February 2021

Citation:

Wang P-P, Ding S-Y, Sun $Y-Y$,

Li Y-H and Fu W-N (2021)

MYCT1 Inhibits the Adhesion and Migration of Laryngeal

Cancer Cells Potentially

Through Repressing

Collagen VI.

Front. Oncol. 10:564733

doi: 10.3389/fonc.2020.564733

\section{MYCT1 Inhibits the Adhesion and Migration of Laryngeal Cancer Cells Potentially Through Repressing Collagen VI}

\author{
Peng-Peng Wang ${ }^{1}$, Si-Yu Ding ${ }^{1}$, Yuan-Yuan Sun ${ }^{1}$, Yun-Hui $\mathrm{Li}^{2 *}$ and Wei-Neng Fu ${ }^{1 *}$ \\ ${ }^{1}$ Department of Medical Genetics, China Medical University, Shenyang, China, ${ }^{2}$ Department of Laboratory Medicine, \\ General Hospital of Northern Theater Command, Shenyang, China
}

MYCT1, a target of c-Myc, inhibits laryngeal cancer cell migration, but the underlying mechanism remains unclear. In the study, we detected differentially expressed genes (DEGs) from laryngeal cancer cells transfected by MYCT1 using RNA-seq (GSE123275). DEGs from head and neck squamous cell carcinoma (HNSCC) were first screened by comparison of transcription data from the Gene Expression Omnibus (GSE6631) and the Cancer Genome Atlas (TCGA) datasets using weighted gene co-expression network analysis (WGCNA). GO and KEGG pathway analysis explained the functions of the DEGs. The DEGs overlapped between GSE6631and TCGA datasets were then compared with ours to find the key DEGs downstream of MYCT1 related to the adhesion and migration of laryngeal cancer cells. qRT-PCR and Western blot were applied to validate gene expression at mRNA and protein levels, respectively. Finally, the cell adhesion, migration, and wound healing assays were to check cell adhesion and migration abilities, respectively. As results, 39 overlapping genes were enriched in the GSE6631 and TCGA datasets, and most of them revealed adhesion function. Thirteen of 39 genes including COL6 members COL6A1, COL6A2, and COL6A3 were overlapped in GSE6631, TCGA, and GSE123275 datasets. Similar to our RNA-seq results, we confirmed that COL6 is a target of MYCT1 in laryngeal cancer cells. We also found that MYCT1 inhibited the adhesion and migration of laryngeal cancer cells via COL6. These indicate that COL6 is a potential target of MYCT1 and participates the adhesion and migration of laryngeal cancer cells, which provides an important clue for further study on how MYCT1 regulating COL6 in laryngeal cancer progression. 


\section{INTRODUCTION}

Head and neck squamous cell carcinoma (HNSCC) is the sixth most common malignancy worldwide with approximately 600,000 new cases each year (1). Laryngeal squamous cell carcinoma (LSCC) is a common type of head and neck cancer. Several genes involved in LSCC tumorigenesis and progression might be potential markers for the diagnosis and treatment of laryngeal cancer (2-7).

Metastasis is the leading cause of death in cancer patients, and movement of tumor cells through the extracellular matrix (ECM) is the first stage of invasion. Thus, invasion is characterized by enhanced cell motility, which is caused by alterations in interactions between cells and between cells and the ECM (8). Collagen VI (COL6), a major ECM protein associated with tumors, is a trimeric molecule consisting of three major polypeptide chains, $\alpha 1$ (VI), $\alpha 2$ (VI), and $\alpha 3$ (VI), which are encoded by COL6A1, COL6A2, and COL6A3, respectively (9).

In the previous study, we first cloned MYCT1 in laryngeal cancer, named as c-Myc target from laryngeal cancer (MTLC) (10). We also found that MYCT1 is a down-regulated and suppress cancer cell migration in laryngeal cancer, suggesting that it acts as tumor suppressor (11). Recently, we obtained lots of differentially expressed genes (DEGs) downstream of MYCT1 in laryngeal cancer by transcriptome sequencing (GSE123275). However, the molecular mechanism of MYCT1 in cancer migration invasion has not been delineated. In the present study, we aim to identify DEGS downstream of MYCT1 through analyzing HNSCC gene expression data from the Gene Expression Omnibus (GEO) and The Cancer Genome Atlas (TCGA) together with ours, and explore their function in the adhesion and migration of laryngeal cancer cells.

\section{MATERIALS AND METHODS}

\section{Cell Lines and Culture Conditions}

The human laryngeal cancer cell line Hep2 was obtained from KeyGen Biotech Company (Nanjing, China). Hep2 cells were cultured in RPMI1640 (GIBCO Thermo Fisher Scientific, MA, USA) supplemented with $10 \%$ fetal bovine serum (FBS, Israel), $100 \mathrm{U} / \mathrm{ml}$ penicillin, and $100 \mathrm{mg} / \mathrm{ml}$ streptomycin (Solarbio, China) in an incubator at $37^{\circ} \mathrm{C}$ with $5 \% \mathrm{CO}_{2}$.

\section{Gene Transfection}

The GV230-MYCT1 overexpression plasmids and small interfering RNAs were purchased from Gene Pharma (Shanghai, China). The siRNA sequences are as follows: siMYCT1: 5'-GCUGUGAACGUCGAAGCAATT-3', siCOL 6A2: 5'-GGAGAGUGCUACAAGGUGAGC-3', siCOL6A3: 5' GGAGGGAGACAUAGUUCUUGG-3' negative control RNA: 5' - UUCUCCGAACGUGUCACGUTT-3'. The Hep2 cells were transfected with the relevant GV230-MYCT1 plasmid and siRNAs using jetPRIME ${ }^{\circledR}$ Transfection Reagent (PolyplusTransfection, Illkirch, France). The cells at 24 or $48 \mathrm{~h}$ post transfection were harvested for further use.

\section{RNA Isolation}

Total RNA was extracted from transfected Hep2 cells using RNAiso plus (Takara, Beijing, China). The RNA concentration was measured by reading the absorbance at $260 / 280 \mathrm{~nm}$.

\section{RNA-Sequencing and Analysis}

RNA sequencing for MYCT1-overexpressing laryngeal cancer cells were carried out by Beijing Genomics Institute (BGI, Shenzhen) with the HiseqTM 4000 PE101 platform. All the gene expression data including those of DEGs were deposited in the GEO database with accession number GSE123275 (https:// www.ncbi.nlm.nih.gov/geo/query/acc.cgi?acc=GSE123275). Then DEGs were selected using a multi-hypothesis test correction for $P$-value methods. Summation of $\log _{2}$-transformed fold changes higher than 1 and $P$-value lower than 0.05 was used as threshold of the DEGs.

\section{Data Collection and Pre-Processing}

GSE6631 HNSCC gene expression data profiles were obtained from the GEO (https://www.ncbi.nlm.nih.gov/geo) database. GSE6631 is a microarray dataset containing 22 pairs of tumor and adjacent non-tumor HNSCC tissues. The clinical characteristics of the samples are included in the original publication (12). The average transcriptional level of each gene was determined by microarray using multiple probes, and annotations labeled by probes were converted to the same gene ID described in the Ensembl database (http://asia.ensembl.org).

RNA-seq data (level 3) from 43 pairs of tumor and adjacent non-tumor HNSCC tissues and clinical information of the corresponding patients were obtained from the TCGA (http:// cancergenome.nih.gov). Each gene was given a sequencing annotation, while was also renamed using their gene ID according to the Ensembl database, and the genes that were not expressed in any of the samples were removed.

\section{Screening for Differentially Expressed Genes in HNSCC}

limma package (http://bioconductor.org/packages/limma) in Rproject was applied to identify DEGs by analyzing differences in their transcription levels between tumor and non-tumor tissues from the GSE6631 dataset. Then, the log2 fold change (log2FC) differential expressions and their statistical significance (Benjamini-Hochberg adjusted $P$-values) were calculated using a linear fit model and empirical Bayes method in the limma package of Bionconductor version 3.8. $|\log 2 \mathrm{FC}|>1$ and adjusted $P$-values $<0.05$ were considered thresholds for screening DEGs in HNSCC. DEGs from GSE6631 were screened out in HNSCC patients and compared with the RNAseq data (level 3) from the TCGA dataset. Based on the transcription levels of DEGs screened out in each sample, the samples were then clustered to evaluate the sample outliers.

\section{Identification of Differentially Expressed Genes Related to HNSCC TNM Stages}

The WGCNA package in R-project was used to identify HNSCC TNM stage-related DEGs. Based on the transcription levels of DEGs from the GSE6631 dataset, a soft threshold $\beta$ value was set 
according to the dynamic threshold to obtain highly related genes, which were clustered to produce signed similarities according to paired Pearson correlation coefficients that were calculated based on gene transcript levels. Scale-free topological overlap matrix (TOM) networks were then generated based on the similarities with 0.25 as the default minimum cluster merge height and 30 as the minimum number of genes to evaluate the correlation among genes in clusters. Based on different correlation values, genes were clustered into different groups marked with different colors that were named as different modules, whereas uncorrelated genes were grouped into the gray module. The related-value and $P$-value of the correlation among genes in each block in the same group were obtained by analyzing their transcription levels when different clinical features were present (13). Based on the transcription levels of DEGs from TCGA datasets, a soft threshold $\beta$ value, TOM, modules, related-value, and $P$-value of the correlation in each block were obtained by the same analysis mentioned above to further confirm the results from the GSE6631 dataset.

A cross-comparison of DEGs in each module was established using the GSE6631 dataset and the TCGA dataset except for the genes in the gray module. The DEGs were then applied to gene ontology (GO) and Kyoto Encyclopedia of Genes and Genomes (KEGG) pathway analyses according to the DAVID (https:// david.ncifcrf.gov) $(14,15)$ and the ClueGO application in Cytoscape version 3.42 (16). In the ClueGO application of Cytoscape, GO based on GO-BiologicalProcess-EBI-QuickGOGOA 12.01.2018 and KEGG based on KEGG 12.01.2018.

\section{qRT-PCR Detection}

RNA was reverse-transcribed to cDNA using a HiScript ${ }^{\circledR}$ II Q RT SuperMix for qPCR (Vazyme Biotech, Nanjing, China). Real-time PCR was performed using TB Green ${ }^{\mathrm{TM}}$ Premix Ex $\mathrm{Taq}^{\mathrm{TM}}$ II (Takara, Beijing, China) under the following conditions: (a) $95^{\circ} \mathrm{C}$ for $30 \mathrm{~s}$, (b) $40 \mathrm{cycles}$ of $95^{\circ} \mathrm{C}$ for $5 \mathrm{~s}$ and $60^{\circ} \mathrm{C}$ for $34 \mathrm{~s}$, and (c) $95^{\circ} \mathrm{C}$ for $15 \mathrm{~s}, 60^{\circ} \mathrm{C}$ for $1 \mathrm{~min}$, and then $95^{\circ} \mathrm{C}$ for $15 \mathrm{~s}$. The relative transcript expression of each gene normalized to glyceraldehyde-3phosphate dehydrogenase (GAPDH) was calculated using the $2^{-\Delta \Delta \mathrm{Ct}}$ method. Primer sequences of the amplified genes are listed in Supplementary File 3.

\section{Western Blot Analysis}

Protein was isolated from cells and subjected to sonication in ice-cold lysis buffer. Denatured protein was separated on sodium dodecyl sulfate-polyacrylamide gel and transferred to a polyvinylidene fluoride (PVDF) membrane (Millipore, MA, USA). After blocking for $2 \mathrm{~h}$ at room temperature, the PVDF membrane was incubated with anti-Collagen Type VI [Proteintech, CHI, USA (17023-1-AP)], anti-MYCT1 [Abcam, Cambridge, UK (ab139945)], and anti- $\beta$-actin [Cloud-Clone Corp. USA (CAB340Mi22)] primary antibodies, followed by incubation with appropriate peroxidase-coupled secondary antibodies. Proteins on the PVDF membrane were stained with ECL (WanleiBio, Shenyang, China) and visualized using ChemiDoc ${ }^{\mathrm{TM}}$ Touch Imaging System (BioRad, CA, USA). The relative protein expression of each gene normalized to $\beta$-actin was calculated based on integrated band density by Image Lab software (Bio-Rad, CA, USA).

\section{Cell Adhesion Assay}

The cell adhesion assay was performed as described below. Briefly, each well of a flat bottom 96-well plate was coated with $50 \mu \mathrm{l}$ of Matrigel $(0.04 \mu \mathrm{g} / \mathrm{ml}$; Corning Life Sciences, NY, USA) in RPMI1640 after the plate was incubated at $37^{\circ} \mathrm{C}$ for $1 \mathrm{~h}$. Each well was blocked with $0.1 \% \mathrm{BSA}$ at $37^{\circ} \mathrm{C}$ for $1 \mathrm{~h}$ followed by three washes in RPMI1640 medium. The cells were harvested by trypsin/ EDTA digestion, washed twice, resuspended in RPMI1640 medium, and labeled by $2 \mu \mathrm{M}$ Calcein AM (KeyGen Biotech Company, Nanjing, China) at $37^{\circ} \mathrm{C}$ for $30 \mathrm{~min}$. Labeled cells $\left(2 \times 10^{4}\right.$ cells/well $)$ were transferred to each well and incubated at $37^{\circ} \mathrm{C}$ for $2 \mathrm{~h}$. Cells in each well were washed three times with RPMI1640 medium to remove nonadherent cells. The fluorescence of adherent cells in each well was measured with a Spark multimode microplate reader (TECAN, Austria) at $490 \mathrm{~nm}$. The percentage of the adherent cells in each well was calculated and normalized to the number of initial input cells.

\section{Cell Migration Assay}

Cell migration was assayed using a 24-well Transwell chamber without Matrigel. Briefly, cells were resuspended in serum-free medium and then seeded in the top chamber. The lower chamber was filled with culture medium containing 10\% FBS. After incubation at $37^{\circ} \mathrm{C}$ for $24 \mathrm{~h}$, cells in the lower chamber were fixed in $4 \%$ paraformaldehyde and stained with hematoxylin and eosin. The migrated cells were visualized and counted in randomly selected fields under a microscope (Leica DM4000 B LED) and analyzed by LAS X software (Leica Microsystems, Wetzlar, GER).

\section{Wound Healing Assay}

Cells were cultured in six-well plates in serum-free medium and were scraped with a $200 \mu \mathrm{l}$ sterile pipette tip at $0 \mathrm{~h}$. Wound healing images were then captured with a Leica DMI3000 B microscope at 0,12 , and $24 \mathrm{~h}$, and then analyzed using LAS AF software (Leica Microsystems, Wetzlar, GER).

\section{Statistical Analysis}

Each experiment was performed at least three times. Data were analyzed by two-tailed Student's $t$-test or one-way analysis of variance using GraphPad Prism 6.01 (GraphPad Software Inc., CA, USA). Data are expressed as the mean \pm standard deviation. $P<0.05$ was considered statistically significant.

\section{RESULTS}

Our RNA-seq results revealed 4,227 DEGs downstream of MYCT1 (GSE123275). In order to find the key DEGs downstream of MYCT1 associated with laryngeal cancer migration and metastasis, we first identified the DEGs in HNSCC by data mining.

\section{Identification of Gene Modules Related to TNM Stage}

Based on the thresholds $|\log 2 \mathrm{FC}|>1$ and adjusted $P$-value $<0.05$, 903 DEGs were found in microarray data from the GSE6631HNSCC dataset whereas 839 in the TCGA-HNSCC RNA-seq 
dataset (Supplementary File 1). In order to set up GSE6631- and TCGA-modules related to TNM stage, scale independence from GSE6631 and TCGA datasets was set at 0.85, soft thresholds for the GSE6631 and TCGA datasets were 12 and 7, respectively, and both datasets obtained higher mean connectivity (Figures 1A, B). Cluster dendrogram results indicated that 903 DEGs from the GSE6631 dataset and 839 DEGs from the TCGA dataset were grouped into 7 and 9 modules, respectively (Figure 1C).
The relationships between DEGs from different modules and clinical features including HNSCC vs. normal tissues and TNM stages were analyzed. As a result, three modules (yellow, blue, and green) from the GSE6631 dataset showed significantly positive correlation with clinical features ( $\mathrm{r}>0.3, P<0.05$ ), whereas three (red, brown, and turquoise) significant negative correlation $(r>-0.3, P<0.05)$. Meanwhile, eight modules (black, red, pink, blue, brown, turquoise, green, and yellow) from the
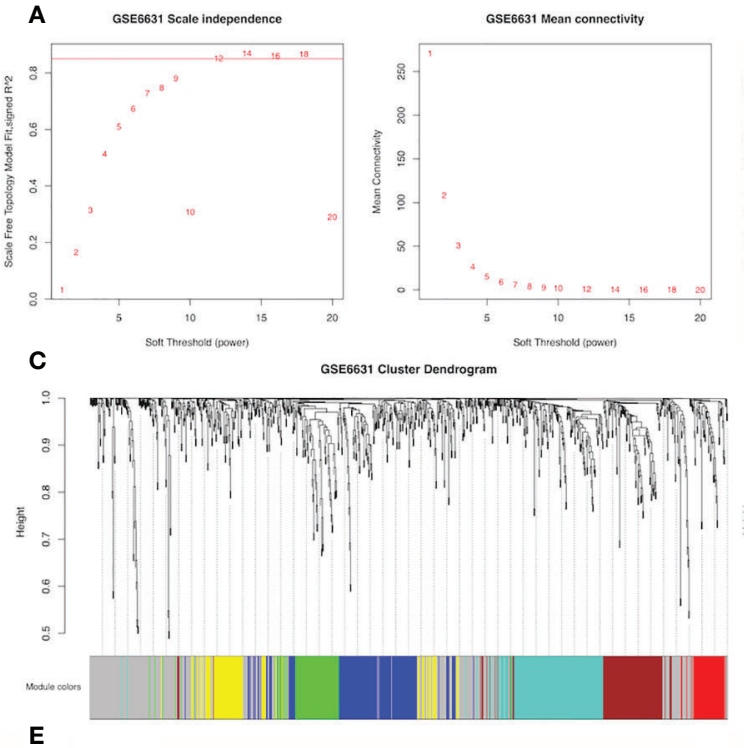

GSE6631 Module-type relationships

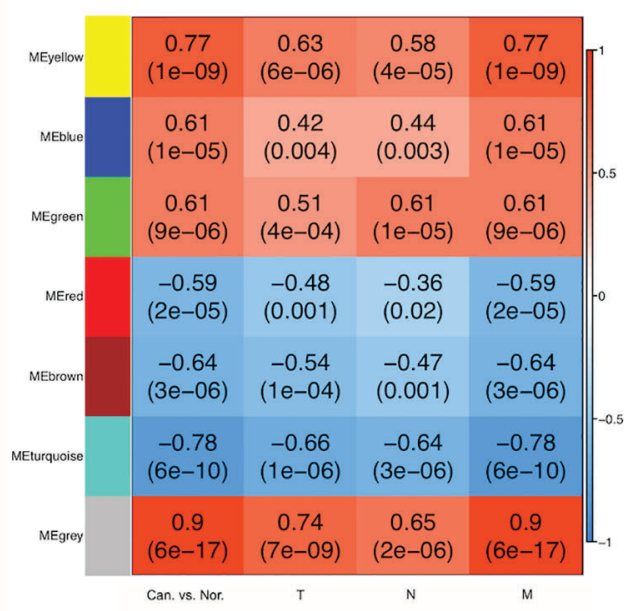

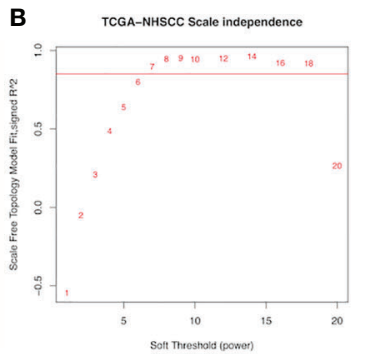

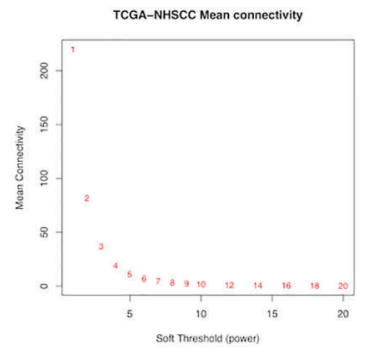

.

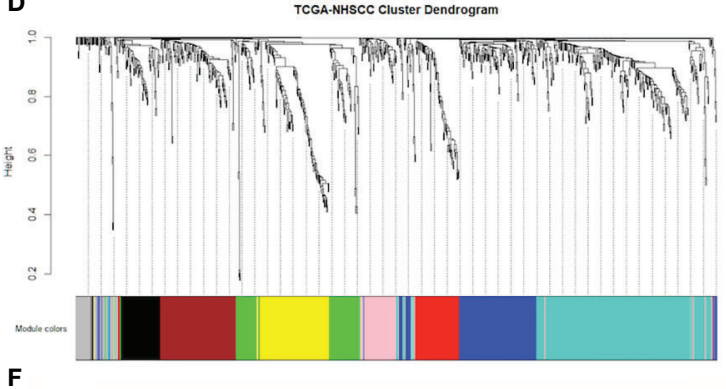

TCGA-HNSCC Module-type relationships

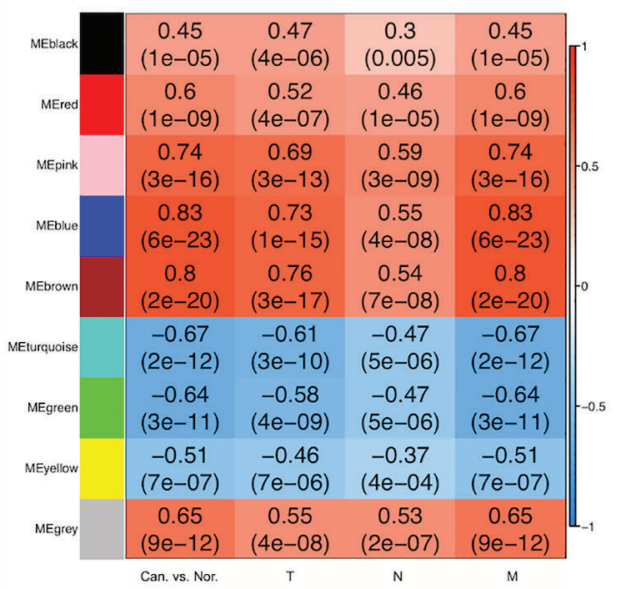

FIGURE 1 | Construction of GSE6631- and TCGA-HNSCC-specific modules using WGCNA. (A) Network topology for different soft-thresholding powers of GSE6631. Left: the scale-free topology fit index (y-axis) in the GSE6631 dataset; right: the mean connectivity (y-axis) as a function of the soft-thresholding power (x-axis) in the GSE6631 dataset. (B) Network topology for different soft-thresholding powers of TCGA-HNSCC. Left: the scale-free topology fit index (y-axis) in the TCGA-HNSCC dataset; right: the mean connectivity (y-axis) as a function of the soft-thresholding power (x-axis) in the TCGA-HNSCC dataset. (C) Branches of the clustering dendrogram from different modules based on differentially expressed genes (DEGs) in GSE6631. Based on the unsupervised hierarchical clustering, GSE6631 DEGs were clustered into seven modules (yellow, blue, green, red, brown, turquoise, and gray). (D) Branches of the clustering dendrogram from different modules based on DEGs in TCGA-HNSCC. Based on the unsupervised hierarchical clustering, TCGA-HNSCC DEGs were clustered into nine modules (black, red, pink, blue, brown, turquoise, green, yellow, and gray). (E) Module-clinical feature relationships in the GSE6631 modules. In the heatmap, red represents a positive correlation, whereas blue represents a negative correlation. The numbers in each block are the related-value and $P$-value of the correlation. (F) Module-clinical feature relationships in TCGA-HNSCC modules. In the heatmap, red represents a positive correlation, and blue represents a negative correlation. The numbers in each block are the related-value and $P$-value of the correlation. 
TCGA dataset revealed significant correlation with clinical features, five of which were positive, whereas three were negative (Figures 1C-F).

\section{Identification of Overlapping Genes Related to the Clinical Features of HNSCC}

Based on gene modules except for the gray ones, a conjoint analysis of all genes between two groups of modules was performed. As a result, all genes in the modules displayed either positive or negative correlations. All positive correlations but not negative ones between modules from each group showed highly repetitive rates (Supplementary File 1), suggesting that negative correlations are not considered in this study. Based on genes in the modules with positive correlations, the most highly repetitive rates were $49 \%$ in the green module from the GSE6631 dataset and $66 \%$ in the red module from the TCGA dataset. Thirty-nine genes having the similar expression pattern between the two modules was observed (Supplementary File 1, Figure 2), which were the focus of the following study.

\section{Enrichment of Signaling Pathways and Functions Points to Adhesion}

GO analysis results revealed that 26 of 39 overlapping genes which were involved in 17 functions (Supplementary File 1, Figure 2A). In the KEGG pathway analysis, 13 of 39 genes were enriched in ECM-receptor interaction, amebiasis, focal adhesion, protein digestion and absorption pathways (Figure 2B). By comparison of the GO terms and the KEGG pathways, most genes including a set of Collagen VI family members were enriched in adhesion function for both GO terms and KEGG

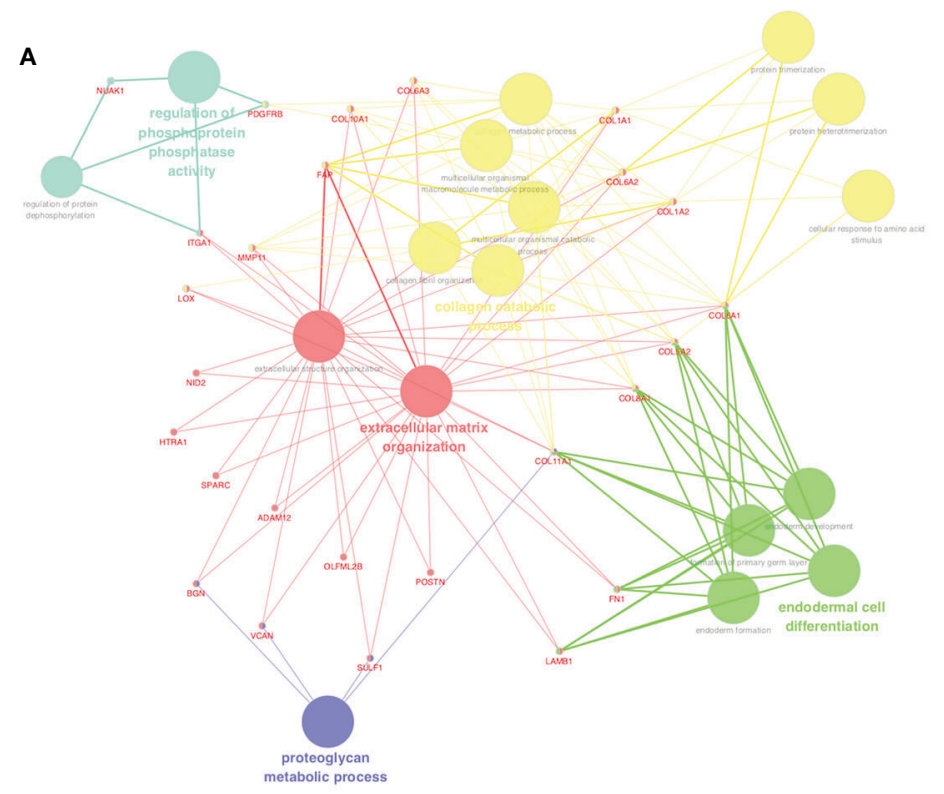

B

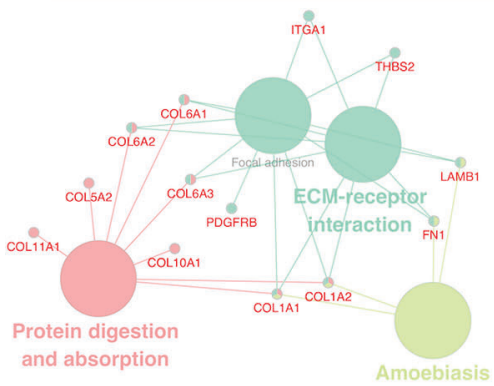

C

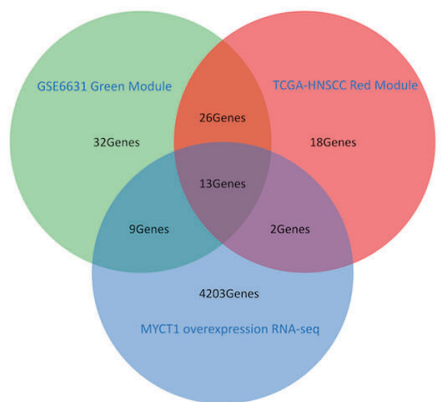

FIGURE 2 | Identification of a functional network and laryngeal cancer-associated DEGs from the red-green overlapping DEGs. (A) GO term interactions among overlapping DEGs. (B) KEGG pathway interactions among overlapping DEGs. (C) Venn diagram of laryngeal cancer associated DEGs downstream of MYCT1 from the GSE123275, GSE6631, and TCGA datasets. The blue circle represents the unique DEGs in the GSE6631 green module. The yellow circle represents the DEGs in the TCGA-HNSCC red modules. Large nodes represent GO terms (A) or KEGG pathways (B), whereas small nodes represent the DEGs. The colors of the nodes represent different GO terms or KEGG pathways in each network diagram. The red circle represents the DEGs downstream of MYCT1 in Hep2 cells. In the middle area, 13 genes were distinguished out of three datasets. 
pathways, which suggest that adhesion should be the focus of the following study (Figures 2A, B).

\section{Collagen VI Family Members are Important Mediators in the MYCT1-Associated Migration of Laryngeal Cancer Cells}

We then compared overlapping genes among the GSE123275, GSE6631, and TCGA datasets. As a result, 13 genes were overlapped, 5 of which belong to collagen family members, including COL6A1, COL6A2, and COL6A3 (Figure 2C, Supplementary File 1), which implies that the 13 genes, especially Collagen VI family members, play importantly potential roles in laryngeal cancer cell migration.

\section{COL6 was Confirmed as a Target Downstream of MYCT1 in Laryngeal Cancer Cells}

In our RNA-seq results, COL6A1, COL6A2, and COL6A3 were downregulated at mRNA levels in MYCT1 overexpressing laryngeal cancer cells (GSE123275). As shown in Figures 3A, B, MYCT1 was significantly decreased and increased both at mRNA and protein levels in laryngeal cancer cells transfected with siMYCT1 and
MYCT1 compared with controls $(P<0.01)$, respectively, suggesting that transfection is successive. At the protein level, Collagen VI was significantly upregulated and downregulated in siMYCT1- and MYCT1-transfected laryngeal cancer cells compared with controls $(P<0.01$, Figure 3B), respectively. At the mRNA level, Knockdown of MYCT1 significantly increased the COL6A1, COL6A2, and COL6A3 expression but MYCT1 decreased the COL6A2, and COL6A3 expression in laryngeal cancer cells $(P<0.01$, Figure 3C), respectively. We also found that both COL6A2 and COL6A3 knockdown significantly recued the effects of MYCT1 silence on their expressions in laryngeal cancer cells $(P<0.01$, Figures 3D, E), respectively. These results confirmed that COL6 is a target of MYCT1 in laryngeal cancer cells.

\section{MYCT1 Inhibits the Adhesion and Migration of Laryngeal Cancer Cells via COL6}

MYCT1 knockdown and overexpression significantly increased and decreased the adhesion, migration and wound healing abilities of the laryngeal cancer cells compared with controls $(P<0.01$,

Figures 4A-C, Supplementary File 2A), respectively. As shown in
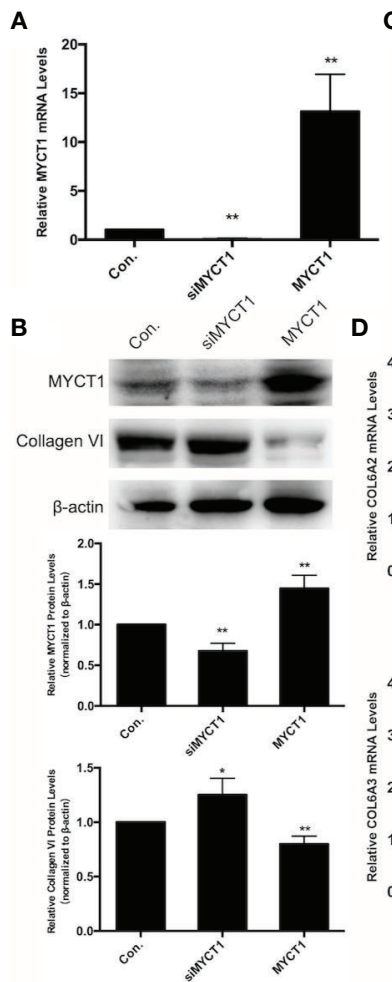

D

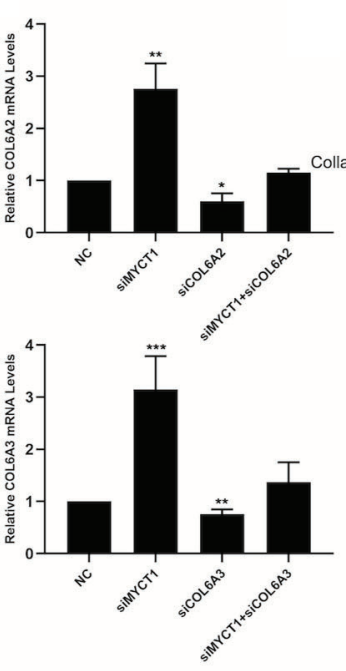

C
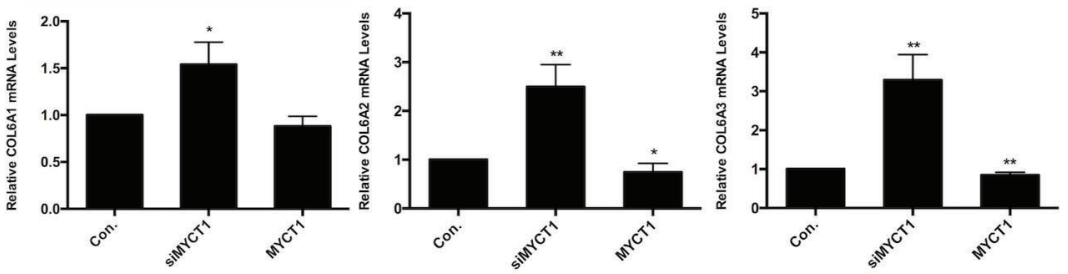

E
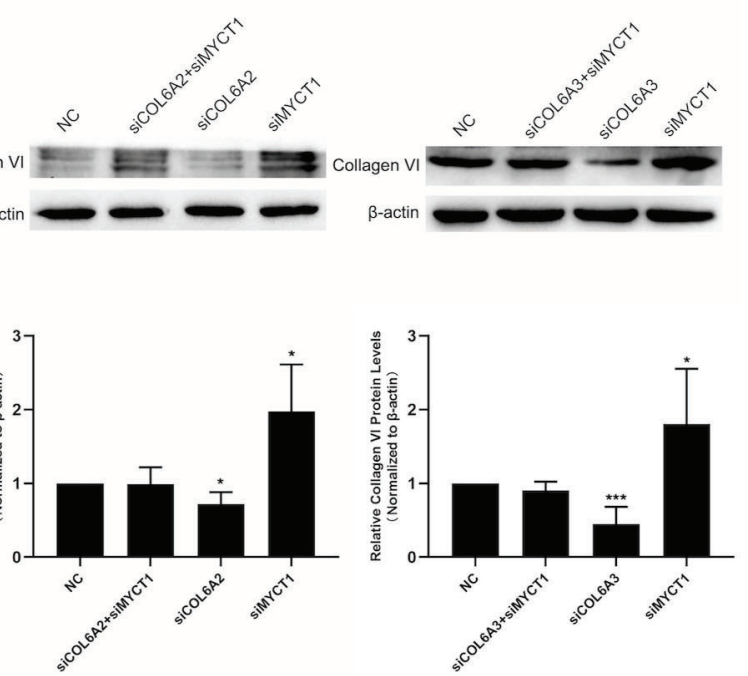

FIGURE 3 | MYCT1 downregulates COL6A1, COL6A2, and COL6A3 gene expression. (A) MYCT1 mRNA level in Hep2 cells transfected with MYCT1 and siMYCT1. (B) Effect of MYCT1 on the levels of COL6A1, COL6A2, and COL6A3 mRNA. The levels of COL6A1, COL6A2, and COL6A3 mRNA were detected by qRT-PCR in Hep2 cells transfected with control, siMYCT1 RNA, or MYCT1. (C) Effect of MYCT1 on the Collagen VI protein level. MYCT1 and Collagen VI protein level in Hep2 cells transfected with MYCT1 and siMYCT1. The Collagen VI protein level was examined by Western blot in Hep2 cells transfected with control, siMYCT1 RNA, or MYCT1. (D) Effects of MYCT1 knockdown on COL6A2 and COL6A3 mRNA expression levels in Hep2 cells by qRT-PCR (E) Effects of MYCT1 knockdown on COL6A2 and COL6A3 protein levels in Hep2 cells by western bolt. Symbols *, ${ }^{\star \star}$ and ${ }^{\star \star \star}$ indicate $\mathrm{P}<0.05, \mathrm{P}<0.01$, and $\mathrm{P}<0.001$, respectively. 

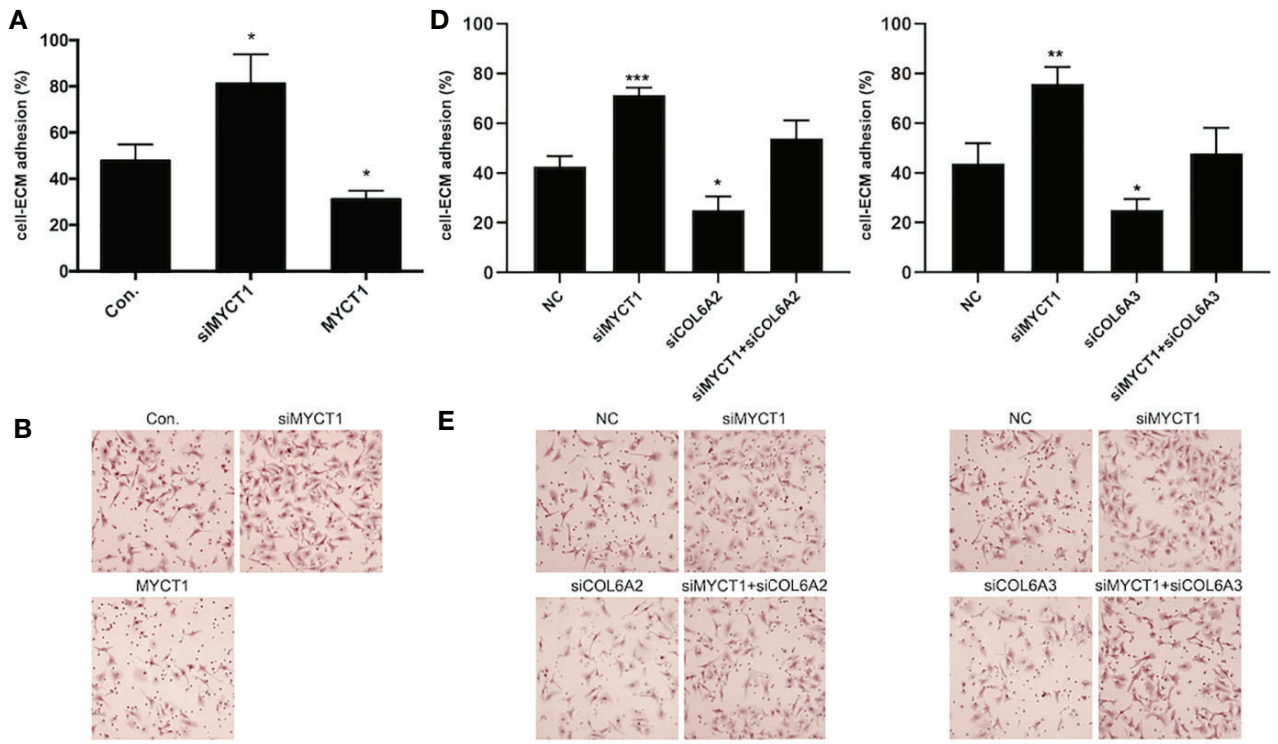

E
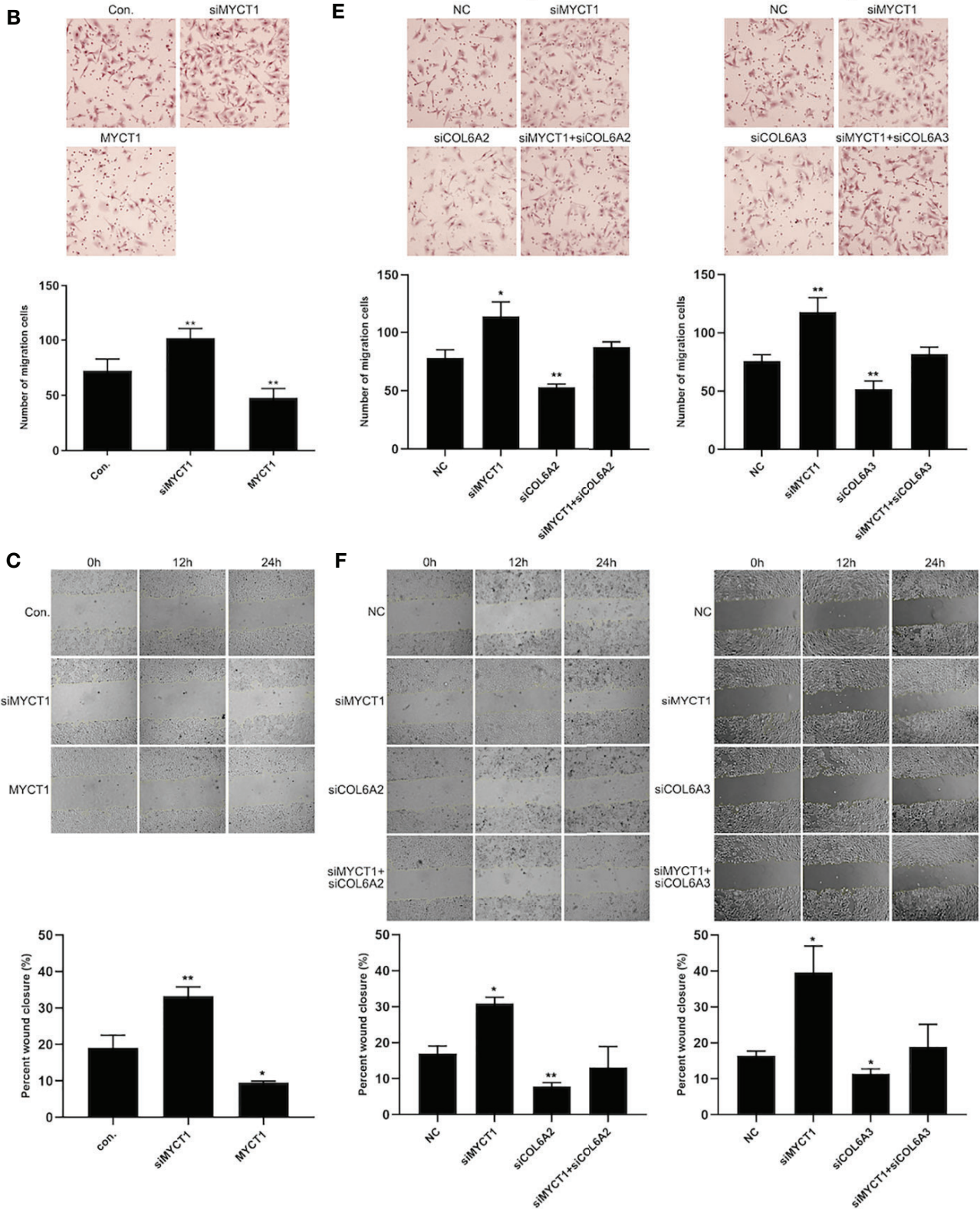

FIGURE 4 | MYCT1 inhibits laryngeal cancer cell migration and adhesion. (A) Effect of MYCT1 on laryngeal cancer cell adhesion by cell adhesion assay. (B) Effect of MYCT1 on laryngeal cancer cell migration according to a Transwell experiment. (C) Effect of MYCT1 on laryngeal cancer cell migration according to a wound healing assay. (D) Effect of MYCT1 knockdown on COL6A2 and COL6A3 in laryngeal cancer cell adhesion by cell adhesion assay. (E) Effect of MYCT1 knockdown on COL6A2 and COL6A3 in laryngeal cancer cell migration according to a Transwell experiment. (F) Effect of MYCT1 knockdown on COL6A2 and COL6A3 in laryngeal cancer cell migration according to a wound healing assay. Symbols * ${ }^{* *}$ and ${ }^{* \star *}$ indicate $P<0.05, P<0.01$, and $P<0.001$, respectively. 
Figures 4D-F and Supplementary File 2A, COL6A2 and COL6A3 knockdown significantly recued the effects of MYCT1 silence on the adhesion, migration and wound healing abilities of the laryngeal cancer cells $(P<0.01)$, respectively. These results indicated that MYCT1 suppresses the adhesion and migration of laryngeal cancer cells via COL6.

\section{DISCUSSION}

Some cellular processes, such as ECM degradation and epithelialto-mesenchymal transition, are essential for cancer metastasis (17). It is generally accepted that cancer invasion and metastasis involve tumor cell adhesion changes, ECM degradation, and cancer cell migration in a proteolytically modified ECM (18). ECM not only acts as a scaffold to which cells are anchored but also plays a significant role in cell function and behavior (19).

COL6 is a major ECM-associated protein in the tumor microenvironment $(20,21)$. COL6 also forms a discrete network of beaded microfilaments, which interact with other ECM molecules and contribute to structural support for cells (22). COL6 is highly expressed in a series of cancer and promotes cancer cell growth and metastasis to directly or indirectly accelerate cancer progression by educating other microenvironmental components $(21,23)$.

WGCNA is an innovative statistical tool used to cluster sets of genes with similar expression patterns and to analyze the correlation between genes and clinical features. WGCNA has been widely used in the analysis of cancer, such as lung (24), breast (25), colorectal (26), liver (27), thyroid (28), and HNSCC (29-31). However, WGCNA-related studies on laryngeal cancer metastasis have seldom been reported.

In this study, we found 903 and 839 DEGs from the GSE6631 and TCGA datasets, respectively, which were grouped into 7 and 9 modules, respectively. Thirty-nine DEGs were present in both datasets, 13 of which, including COL6A1, COL6A2, and COL6A3, were enriched in ECM-receptor interaction, amebiasis, focal adhesion, protein digestion, and absorption pathways. In MYCT1-overexpressing laryngeal cancer cells, another 13 DEGs (GSE123275) were overlapped in the GSE6631 and TCGA datasets, where COL6A1, COL6A2, and COL6A3 were also present. This implies that COL6A1, COL6A2, and COL6A3 are crucial in laryngeal cancer.

In our previous study, RNA-seq detection showed that the significantly low expression of COL6A1, COL6A2, and COL6A3 in MYCT1-overexpressing laryngeal cancer cells that was confirmed in the study, which suggests that the three COL6 members are potential targets downstream of MYCT1 in laryngeal cancer. However, the mechanism is still unclear.

In the study, we also found that MYCT1 inhibits laryngeal cancer cell adhesion and migration and the effects are rescued by Collagen VI. These findings indicate that MYCT1 participates in the regulation of laryngeal cancer cell adhesion and migration at least via downregulating the COL6 gene expression. Previously, we discovered that MYCT1 suppresses laryngeal cancer cell migration through the CREB/MYCT1/NAT10 axis (32). But
MYCT1-mediated laryngeal cancer cell adhesion has not been reported. Therefore, we plan to focus on the following two issues in future studies: 1) regulatory mechanism of MYCT1 on COL6; 2) molecular mechanism of COL6 in laryngeal cancer cell adhesion and migration.

\section{CONCLUSIONS}

We first found that a set of DEGs such as COL6A1, COL6A2, and COL6A3 are probably involved in the progression laryngeal cancer and MYCT1 inhibits the adhesion and migration of laryngeal cancer cells potentially through repression of COL6, suggesting that COL6 is a target downstream of MYCT1 and contributes to the progression of laryngeal cancer.

\section{DATA AVAILABILITY STATEMENT}

The datasets presented in this study can be found in online repositories. The names of the repository/repositories and accession number(s) can be found below: https://www.ncbi.nlm. nih.gov/geo/, GSE6631 and GSE123275; http://cancergenome. nih.gov, RNA-seq data (level 3).

\section{AUTHOR CONTRIBUTIONS}

Conceived and designed the experiments, P-PW, W-NF. Performed the experiments analyzed the data, P-PW, S-YD. Contributed reagents, Y-YS. Materials and analysis tools, Y-HL. Contributed on the writing of the manuscript P-PW, W-NF. All authors contributed to the article and approved the submitted version.

\section{FUNDING}

This work was supported by the National Natural Science Foundation of China (81372876), Liaoning Science and Technology Project (2017020201-301, LQNK201726).

\section{SUPPLEMENTARY MATERIAL}

The Supplementary Material for this article can be found online at: https://www.frontiersin.org/articles/10.3389/fonc.2020. 564733/full\#supplementary-material

Supplementary File 1 | List of differentially expressed genes in different datasets.

Supplementary File 2 | High resolution of Figures 4C and 4D

Supplementary File 3 | Primer sequences used in the study. 


\section{REFERENCES}

1. Bray F, Ferlay J, Soerjomataram I, Siegel RL, Torre LA, Jemal A. Global cancer statistics 2018: GLOBOCAN estimates of incidence and mortality worldwide for 36 cancers in 185 countries. CA Cancer J Clin (2018) 68(6):394-424. doi: $10.3322 /$ caac. 21492

2. Chen $\mathrm{D}$, Gong $\mathrm{L}$, Jiang $\mathrm{Q}$, Wang $\mathrm{X}$, Zhang B. Interaction between MLL3 genetic polymorphisms, smoking, and alcohol drinking in laryngeal cancer: a case-control study. Cancer Med (2016) 5(3):527-33. doi: 10.1002/cam4.589

3. Wong N, Khwaja SS, Baker CM, Gay HA, Thorstad WL, Daly MD, et al. Prognostic microRNA signatures derived from The Cancer Genome Atlas for head and neck squamous cell carcinomas. Cancer Med (2016) 5(7):1619-28. doi: $10.1002 /$ cam 4.718

4. Ben-Dayan MM, Ow TJ, Belbin TJ, Wetzler J, Smith RV, Childs G, et al. Nonpromoter methylation of the CDKN2A gene with active transcription is associated with improved locoregional control in laryngeal squamous cell carcinoma. Cancer Med (2017) 6(2):397-407. doi: 10.1002/cam4.961

5. Mo BY, Li GS, Huang SN, He WY, Xie LY, Wei ZX, et al. The underlying molecular mechanism and identification of transcription factor markers for laryngeal squamous cell carcinoma. Bioengineered (2021) 12(1):208-24. doi: 10.1080/21655979.2020.1862527

6. Chen L, Xu Z, Zhao J, Zhai X, Li J, Zhang Y, et al. H19/miR-107/HMGB1 axis sensitizes laryngeal squamous cell carcinoma to cisplatin by suppressing autophagy in vitro and in vivo. Cell Biol Int (2020). doi: 10.1002/cbin.11520

7. Cao Y, Zhan GR, Luo X, Yang Y. LncRNA PART1 promotes lung squamous cell carcinoma progression via miR-185-5p/Sixl axis. Hum Exp Toxicol (2020), 960327120979032. doi: 10.1177/0960327120979032.

8. Paolillo M, Schinelli S. Extracellular Matrix Alterations in Metastatic Processes. Int J Mol Sci (2019) 20(19):4947. doi: 10.3390/ijms20194947

9. Mohassel P, Foley AR, Bonnemann CG. Extracellular matrix-driven congenital muscular dystrophies. Matrix Biol (2018) 71-72:188-204. doi: 10.1016/j.matbio.2018.06.005

10. Qiu G, Qiu G, Xu Z, Huang D, Gong L, Li C, et al. Cloning and characterization of MTLC, a novel gene in 6q25. Zhonghua Yi Xue Yi Chuan Xue Za Zhi (Chinese) (2003) 20(2):94-7.

11. Fu S, Guo Y, Chen H, Xu ZM, Qiu GB, Zhong M, et al. MYCT1-TV, a novel MYCT1 transcript, is regulated by c-Myc and may participate in laryngeal carcinogenesis. PloS One (2011) 6(10):e25648. doi: 10.1371/journal.pone.0025648

12. Kuang J, Zhao M, Li H, Dang W, Li W. Identification of potential therapeutic target genes and mechanisms in head and neck squamous cell carcinoma by bioinformatics analysis. Oncol Lett (2016) 11(5):3009-14. doi: 10.3892/ ol.2016.4358

13. Langfelder $P$, Horvath S. Eigengene networks for studying the relationships between co-expression modules. BMC Syst Biol (2007) 1:54. doi: 10.1186/17520509-1-54

14. da Huang, W, Sherman BT, Lempicki RA. Systematic and integrative analysis of large gene lists using DAVID bioinformatics resources. Nat Protoc (2009) 4 (1):44-57. doi: 10.1038/nprot.2008.211

15. Huang da W, Sherman BT, Lempicki RA. Bioinformatics enrichment tools: paths toward the comprehensive functional analysis of large gene lists. Nucleic Acids Res (2009) 37(1):1-13. doi: 10.1093/nar/gkn923

16. Bindea G, Mlecnik B, Hackl H, Charoentong P, Tosolini M, Kirilovsky A, et al. ClueGO: a Cytoscape plug-in to decipher functionally grouped gene ontology and pathway annotation networks. Bioinformatics (2009) 25(8):1091-3. doi: 10.1093/bioinformatics/btp101

17. Ghasemi A, Saeidi J, Azimi-Nejad M, Hashemy SI. Leptin-induced signaling pathways in cancer cell migration and invasion. Cell Oncol (Dordr) (2019) 42 (3):243-60. doi: 10.1007/s13402-019-00428-0

18. Yuzhalin AE, Lim SY, Kutikhin AG, Gordon-Weeks AN. Dynamic matrisome: ECM remodeling factors licensing cancer progression and metastasis. Biochim Biophys Acta Rev Cancer (2018) 1870(2):207-28. doi: 10.1016/j.bbcan.2018.09.002

19. Cramer MC, Badylak SF. Extracellular Matrix-Based Biomaterials and Their Influence Upon Cell Behavior. Ann BioMed Eng (2020) 48(7):2132-53. doi: 10.1007/s10439-019-02408-9

20. Bernardi ,P, Bonaldo P. Dysfunction of mitochondria and sarcoplasmic reticulum in the pathogenesis of collagen VI muscular dystrophies. Ann N Y Acad Sci (2008) 1147:303-11. doi: 10.1196/annals.1427.009

21. Chen P, Cescon M, Bonaldo P. Collagen VI in cancer and its biological mechanisms. Trends Mol Med (2013) 19(7):410-7. doi: 10.1016/j.molmed. 2013.04.001

22. Cescon M, Gattazzo F, Chen P, Bonaldo P. Collagen VI at a glance. J Cell Sci (2015) 128(19):3525-31. doi: 10.1242/jcs.169748

23. Willumsen N, Bager C, Karsdal MA. Matrix Metalloprotease Generated Fragments of Type VI Collagen Have Serum Biomarker Potential in Cancer A Proof of Concept Study. Transl Oncol (2019) 12(5):693-8. doi: 10.1016/ j.tranon.2019.02.004

24. Ling B, Liao X, Huang Y, Liang L, Jiang Y, Pang Y, et al. Identification of prognostic markers of lung cancer through bioinformatics analysis and in vitro experiments. Int J Oncol (2020) 56(1):193-205. doi: 10.3892/ ijo.2019.4926

25. Li J, Liu C, Chen Y, Gao C, Wang M, Ma X, et al. Tumor Characterization in Breast Cancer Identifies Immune-Relevant Gene Signatures Associated With Prognosis. Front Genet (2019) 10:1119. doi: 10.3389/fgene.2019.01119

26. Xi Y, Yuefen P, Wei W, Quan Q, Jing Z, Jiamin X, et al. Analysis of prognosis, genome, microbiome, and microbial metabolome in different sites of colorectal cancer. J Transl Med (2019) 17(1):353. doi: 10.1186/s12967-0192102-1

27. Kong J, Wang T, Zhang Z, Yang X, Shen S, Wang W. Five Core Genes Related to the Progression and Prognosis of Hepatocellular Carcinoma Identified by Analysis of a Coexpression Network. DNA Cell Biol (2019) 38(12):1564-76. doi: 10.1089/dna.2019.4932

28. Ao ZX, Chen YC, Lu JM, Shen J, Peng LP, Lin X, et al. Identification of potential functional genes in papillary thyroid cancer by co-expression network analysis. Oncol Lett (2018) 16(4):4871-8. doi: 10.3892/ol.2018.9306

29. Guo YZ, Sun HH, Wang XT, Wang MT. Transcriptomic analysis reveals key lncRNAs associated with ribosomal biogenesis and epidermis differentiation in head and neck squamous cell carcinoma. J Zhejiang Univ Sci B (2018) 19 (9):674-88. doi: 10.1631/jzus.B1700319

30. Zhao G, Fu Y, Su Z, Wu R. How Long Non-Coding RNAs and MicroRNAs Mediate the Endogenous RNA Network of Head and Neck Squamous Cell Carcinoma: a Comprehensive Analysis. Cell Physiol Biochem (2018) 50 (1):332-41. doi: 10.1159/000494009

31. Zeng H, Li H, Zhao Y, Chen L, Ma X. Transcripto-based network analysis reveals a model of gene activation in tongue squamous cell carcinomas. Head Neck (2019) 41(12):4098-110. doi: 10.1002/hed.25952

32. Zhang ZX, Zhang WN, Sun YY, Li YH, Xu ZM, Fu WN. CREB promotes laryngeal cancer cell migration via MYCT1/NAT10 axis. Onco Targets Ther (2018) 11:1323-31. doi: 10.2147/ott.s156582

Conflict of Interest: The authors declare that the research was conducted in the absence of any commercial or financial relationships that could be construed as a potential conflict of interest.

Copyright (C) 2021 Wang, Ding, Sun, Li and Fu. This is an open-access article distributed under the terms of the Creative Commons Attribution License (CC BY). The use, distribution or reproduction in other forums is permitted, provided the original author(s) and the copyright owner(s) are credited and that the original publication in this journal is cited, in accordance with accepted academic practice. No use, distribution or reproduction is permitted which does not comply with these terms. 